\title{
INFRARED ROTATION PLANE RADAR CHANGE DETECTION
}

\author{
Wang Yu, Li Jiatian, Zhang Wenjing, Xiao Yi, Liu Peng, Zhang Sijia* \\ Institute of land and Resources Engineering, Kunming University of Science and Technology, Kunming, China - \\ wyvirtues@163.com
}

KEY WORDS: Infrared Rotation Plane Radar, Change Detection, Depth Information, Mixed Gaussian, Background Modeling

\begin{abstract}
:
The existing change detection method mainly stays on the pixel-level, which is very susceptible to light, shadow, etc. And the complex calculation and analysis for each pixel reduce the detection efficiency. Moreover, there is no modeling determination method to initialize standard deviation of each element for existing mixed Gaussian background modeling methods. In this paper, an improved mixed Gaussian background modeling method is proposed, with the use of infrared rotation plane radar. The relationship between the corrected standard deviation of distance and the detection intensity is used to establish the curve of standard deviation of distance with detected intensity. For each data point, the standard deviation is initialized by the value estimated by the change curve, and the detected distance is used to establish the Gaussian mixture background model. The detection effect of the method is discussed and compared with the traditional Gauss background modeling in the experiment, the result shows that it has certain advantages in processing speed, adaptability to change background and accuracy of change detection.
\end{abstract}

\section{INTRODUCTION}

Change detection is one branch of image processing and pattern recognition as well as one of the hotspots in the research of machine vision. With the development of moving object detection technology and its important uses in video surveillance, coding, and content based retrieval, etc. It has got more and more extensive research and application (Radke et al., 2005a; Liu et al., 2012a; Zhen et al., 2008a).

There are three commonly used methods for change detection: optical flow method, frame difference method and background difference method. Among them, the background difference method is the most used method in change detection in recent years (Bharti, 2013a). Background difference method initializes the background model at the training stage, and updates model parameters in real time, then the change targets are extracted by comparing with the background model at the test stage. In many cases, we need to quickly detect the change area and analyze the changing area on demand. The background modeling method based on the mixed Gauss model, as shown in previous work (Stauffer, 1999a), is one of the most successful methods. The method uses multiple Gauss distributions to establish the background model, and constantly adjusts the composition of the Gauss distribution in the background model through background updating, so that it has certain adaptability to scenes. Wang et al. (2014a) have studied the SAR image change detection method based on Gauss hybrid model and Hou et al. (2013a) have researched on infrared small target detection method based on high pass filtering and image enhancement. However, in the actual change detection process, there are still some problems to be solved in the mixed Gauss model. 1) A number of fixed Gauss distributions are established at each pixel point, which will consume a large number of system resources in processing. 2) When the illumination mutation occurs, it is prone to cause a large area of false positives (Akula et al., 2014a; Zhou et al., 2013a; Shimada et al., 2016a).For high-density and complex monitoring occasions, traditional video-based change detection is difficult to meet the application requirements. Although high speed ball camera is suitable for the monitoring of large areas and active targets, it is complex and expensive. It is feasible to detect the environment, using infrared rotating plane radar to convert the distance of the photographed scene to generate depth information.

In this paper, the change detection is abstracted to a section by using the infrared plane radar to simplify the change detection. Firstly, filter the original data using the singular data filtering method based on Pauta criterion. Then, use polynomial curve fitting to establish the noise model of data to initialize Gauss model and the improved Gauss model is used to establish the background model. Lastly, compare the current period data with the model, and the changing area is separated.

\section{IMPROVED GAUSS BACKGROUND MODELING METHOD}

The existing methods of change detection are generally based on remote sensing images or videos. Although these methods are relatively mature, it's easy to be affected by lights, shadows, etc. Therefore, a method of change detection using laser echo of infrared plane radar is proposed, which still follow the general steps of the background difference method. The main problem of the background difference method is the establishment of the background model. How to construct an efficient and practical background model to obtain a reliable background image is the key point of the background difference method.

\subsection{Characteristics of Infrared Laser Radar Ranging}

The product is based on the ToF principle, cooperating with unique optics, electricity, design to achieve stable, accurate, high sensitive and high-speed distance measurement. ToF is the abbreviation of "Time of Flight Technology". That is to say, the sensor sends out the modulated near infrared light, then the light

\footnotetext{
Corresponding author.

Li Jiatian-1jtwcx@163.com
} 


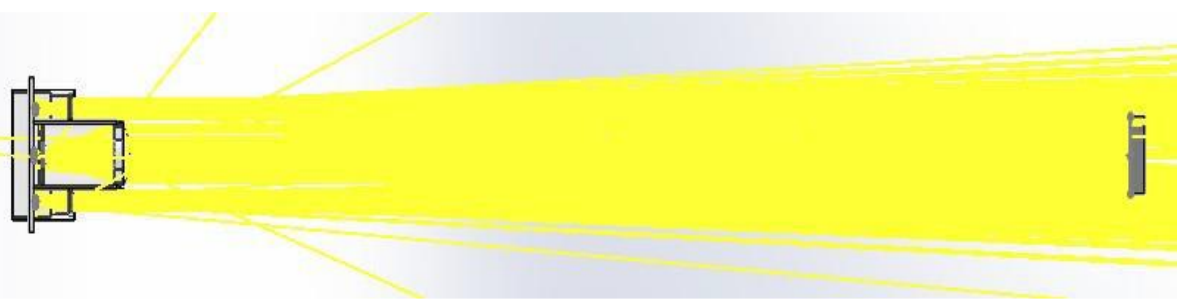

Figure 1. Light path diagram of optical simulation

is reflected by the object, the sensor is used to calculate the time difference or phase difference of the emission and reflection to convert the distance from the scene to help produce the depth of environmental information. The optical simulation optical path is as figure 1 .

The infrared plane radar detects the environment to build a map during the rotation process. The detection range is up to $15 \mathrm{~m}$, and it can also support omni-directional scanning to sense obstructions. The radar is not affected by the color and material of the object in the scanning process, and it works steadily not only in the light but also in the dark. It can also quickly perceive and identify obstacles that suddenly appear in the environment. When each point is scanned, the distance (Dist), angle (Ang), Strength (Strgth) and other information are obtained.

\subsection{Mixed Gauss Background Modeling}

Each Gauss distribution can represent a background scene, and multiple Gauss models can be used to simulate the multimodal situation in a complex scene. A mixed Gauss model of $K$ Gauss distribution is used to represent the probability distribution of the same pixel in the time domain, if the pixel value of a pixel in the image at different times in $t$ is represented as: $\left\{X_{1}, X_{2}, \cdots, X_{t}\right\}$, the probability of the pixel value of the pixel

$$
P\left(X_{t}\right)=\sum_{i=1}^{K} \omega_{i, t} f\left(X_{t}, \mu_{i, t}, \sum_{i, t}\right)
$$

where $\omega_{i, t}, \mu_{i, t}$, and $\sum_{i, t}$ represent the weight of ith Gauss distribution at $\mathrm{t}$, the mean vector and the covariance matrix, $f$ represents Gaussian probability-density function:

$$
f\left(X_{t}, \mu_{i, t}, \quad \sum_{i, t}\right)=\frac{1}{(2 \pi)^{\frac{n}{2}}\left|\sum_{i, t}\right|^{\frac{1}{2}}} e^{-\frac{1}{2}\left(X_{t}-\mu_{i, t}\right)^{T} \sum_{i, t}^{-1}\left(X_{t}-\mu_{i, t}\right)}
$$

where $n$ represents the dimension of $X_{t}$. The $K$ Gauss distributions are arranged in descending order of $\omega_{i, t} / \sigma_{i, t}$.

A new period of observation $X_{t}$ is matched one by one with the well-arranged $K$ Gauss distribution, the matching detection expression is expressed as: $\left|X_{t}-\mu_{i, t}\right|<\lambda \sigma_{i, t-1}$. If the ith distribution of Gauss is matched with $X_{t}$, formula (4) to (6) are executed.

$$
\begin{gathered}
M_{i, t}=\left\{\begin{array}{l}
1, \text { match } \\
0, \text { otherwise }
\end{array}\right. \\
\omega_{i, t}=(1-\alpha) \omega_{i, t-1}+\alpha M_{i, t} \\
\mu_{i, t}=(1-\rho) \omega_{i, t-1}+\rho X_{t} \\
\sigma_{i, t}^{2}=(1-\rho) \sigma_{i, t-1}^{2}+\rho\left(X_{t}-\mu_{i, t}\right)^{T}
\end{gathered}
$$

where $\alpha$ represents the update rate of the weight, $\rho$ represents the update rate of the mean and variance, $\rho=\alpha f\left(X_{i}, \mu_{i}, \sum_{i}\right)$. If it isn't matched, formula (4) and (5) are used to update the weight and the mean, while the variance remain unchanged. If $X_{t}$ isn't matched with all the $K$ Gauss distribution, a new Gauss distribution is introduced, put the last. $X_{t}, \sigma_{\text {init }}$ and $\omega_{\text {init }}$ are the mean, standard deviation and weight of the new Gauss distribution, respectively. The weight of each Gauss are normalized after the completion of the update to make $\sum_{i=1}^{S} \omega_{i, t}$ equal to 1 . The $K$ Gauss distribution is rearranged in descending order of $\omega_{i, t} / \sigma_{i, t}$, and the detection is used to select the first $S$ Gauss, which are taken as the background model, the detection is expressed as

$$
\sum_{i=1}^{S} \omega_{i, t}>T
$$

where $T$ is the threshold for determining the number of the Gauss distribution in the background model. During change detection, if $X_{t}$ is matched with any one of the $S$ Gauss distribution, the pixel belongs to the background. Otherwise, it belongs to the change points.

\subsection{Preprocessing}

In general, only if the length of the target object is greater than the length of the detection range, the output data of the radar can be trusted. When the length of the detection object is less than the detection range, the radar output data fluctuates and the error increases. When the detection object exceeds the effective detection scope, invalid points will occur. If these outliers can be eliminated, the results of the measurement will be more consistent with the objective situation (Sun, 2013a). Therefore, data preprocessing is needed to filter out invalid points so as not to affect subsequent detection. The main error of the radar data acquisition is caused by the external conditions, such as the distance, the length and the complexity of the detection objects. So, the data filtering method based on singular Pauta criterion is adopted here to process the original data.

When the number of measurements $N$ is enough and the measurement obeys the normal distribution, Pauta criterion is conducted in following steps:

1. The arithmetic mean values from $X_{i}$ to $X_{N}$ of the $N$ measurements is calculated as

$$
\bar{X}=\frac{1}{N} \sum_{i=1}^{N} X_{i}
$$

2. Residual error of each item $V_{i}$ is calculated as

$$
V_{i}=X_{i}-\bar{X}
$$

3. The standard deviation $\sigma$ is calculated as

$$
\sigma=\sqrt{\left(\sum_{i=1}^{N} V_{i}^{2}\right) /(N-1)}
$$

4. If expression (11) is satisfied, $X_{i}$ is considered as a bad value 
and is eliminated.

$$
V_{i}>3 \sigma
$$

\subsection{Adaptive Initialization of Standard Deviation}

In existing mixed Gauss model with the same initial standard deviation, the discreteness of each point is different, so the robustness of the model is poor. Evangelio et al. (2014a) have estimated the standard deviation of the previous distribution using the median of absolute deviation. In order to make the model parameters and the standard deviation more consistent with the degree of discretization of each data, it is necessary to determine the variation curve of the corrected distance standard difference $(\sigma)$ with the strength, according to the established change curve, the initial standard deviation for each point is defined as:

$$
\sigma_{i}=\hat{\sigma}_{i}
$$

The curve fitting method is used here to establish the curve of standard deviation of distance with detected intensity, which is a fairly simple method.

The training set is composed of the $\mathrm{N}$ observations of the intensity s, which can be expressed as $S=\left\{s l, \cdots, s_{n}\right\}^{T}$, accompanied by the calculated values of the corresponding corrected distance standard deviation, which are calculated from the previous 30 period data. Assuming that the standard deviation of correction and intensity S satisfy the relation: $\hat{\sigma}=f(s)$. The fitting model is expressed as

$$
f(s, w)=\omega_{0}+\omega_{1} s+\cdots+\omega_{n} s^{N}=\sum_{i=0}^{M} w_{i} s^{i}
$$

which is called polynomial fitting. The values of the coefficients can be determined by adjusting the polynomial function to fit the training data, which can be achieved by the least square fitting method, that's to obtain the minimization of $E(\omega)$.

$$
E(\omega)=\frac{1}{2} \sum_{i=1}^{N}\left\{f\left(s_{n}, \omega\right)-\sigma\right\}^{2}
$$

where factor $\frac{1}{2}$ is added for the convenience of the subsequent operation, $M$ represents the order. We can solve the curve fitting problem by choosing $\omega$ to make $E(\omega)$ as small as possible. Since the error function is the quadratic function of coefficient $\omega$, the first derivative to the coefficient is a linear function of $\omega$, so the minimum value of the error function has a unique solution $\omega^{*}$, which can be obtained by analytical method. The final polynomial function is given by function $f\left(s_{n}, \omega^{*}\right)$. As for the selection of each $M$, formula (14) is used to calculate $E\left(\omega^{*}\right)$ of the training set. Sometimes it is more convenient to use the root mean square error (RMS), which is defined as follow:

$$
E_{R M S}=\sqrt{2 E\left(\omega^{*}\right) / N}
$$

where $2 E\left(\omega^{*}\right)$ is divided by $\mathrm{N}$ to allow us to compare different sizes of data sets on the same basis, and taking the square root of $2 E\left(\omega^{*}\right) / N$ ensures that $E_{R M S}$ is measured with the same size and unit as the target variable $\sigma$ is. The function will show a violent shock, if $M$ is too large or too small. Here, $M$ is selected to be 9 by training.

We establish the changing function of standard deviation of correction following the strength changes, and figure 2 shows the variation curve.

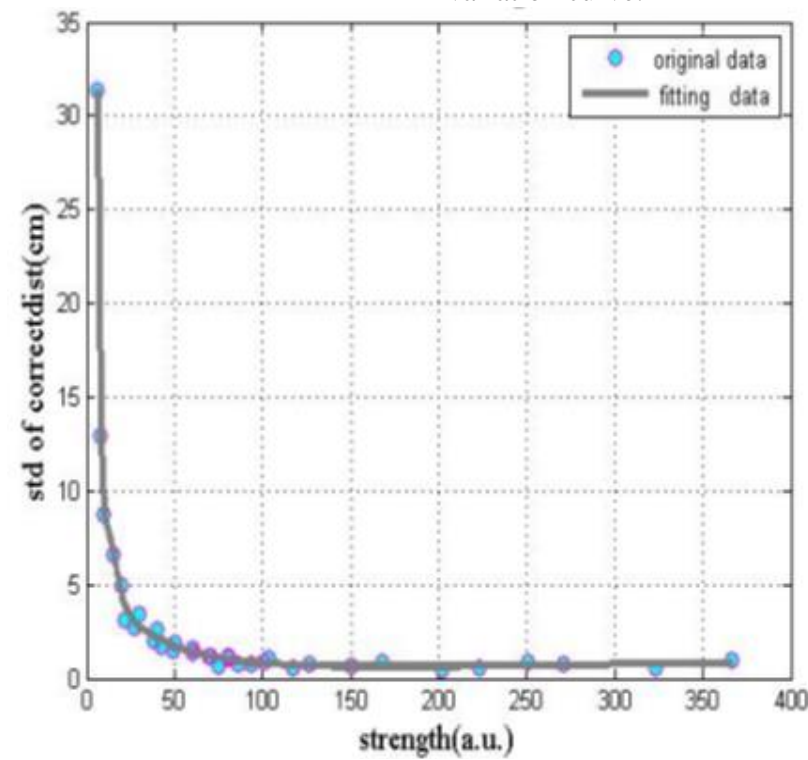

Figure 2. The relationship between standard deviation of correction and the strength

\begin{tabular}{ccccccc}
\hline data type & \multicolumn{4}{c}{ video data } & \multicolumn{2}{c}{ radar data } \\
\hline data & video1 & vidoe2 & video3 & video4 & dataset1 & dataset2 \\
\hline speed & 27.8 & 26.9 & 27.5 & 27.4 & 19.4 & 18.8 \\
average & \multicolumn{2}{c}{$27.4(\mathrm{~ms} / \mathrm{f})$} & \multicolumn{2}{c}{$19.1(\mathrm{~ms} / \mathrm{T})$} \\
\hline
\end{tabular}

Table 3. Processing speed of this method and the traditional method 


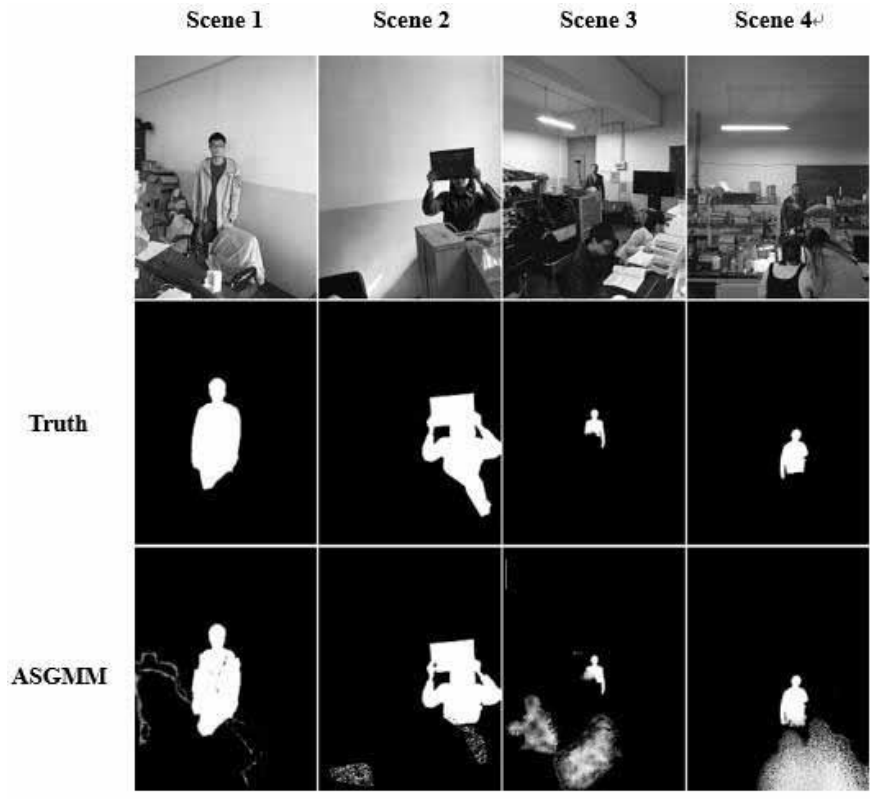

Figure 4. Detection result of ASGMM

\subsection{Update of Background Model}

The convergence rate of the mixed Gauss model is slow and the adaptability to the background changes is weak (Power et al., 2002a). For example, when a moving object stays for a long time to block the background, the original background model is changed. Thus, it is necessary to update the scene in real time. Huang et al. (2010a) have adjusted the number of models online and adjusts the learning rate to adapt to the background changes and maintain the stability of the background. The correlation coefficient is used in previous work ( $\mathrm{Li}$ et al., 2007a) to judge whether the two images are related to reach the goal of matching. And in this paper, the correlation coefficient of current value $d$ and the mean value $u$, obtained from the training cycles, is calculated to judge the correlation between the current frame and the background model, from which we can determine whether the scene should be updated. Such update method can not only make necessary background updates but also avoid the memory waste caused by real time updates.

$$
\operatorname{cov}(d, u)=\frac{\sum_{i=1}^{n}\left(d_{i}-\bar{d}\right)\left(u_{i}-\bar{u}\right)}{\sqrt{\sum_{i=1}^{n}\left(d_{i}-\bar{d}\right)^{2} \sum_{i=1}^{n}\left(u_{i}-\bar{u}\right)^{2}}}
$$

where $\bar{d}$ represents the mean value of all the value $d_{i}$ in current frame, and $\bar{u}$ expresses the mean value of $u_{i}$. If $\operatorname{cov}(d, u)<T$, the Gauss model need to be retrained. In order to reduce computation load, descending sampling is used here to decrease points. And we choose the drop sampling coefficient as 3 , that is, taking a point at every 3 points. After times of training, the threshold $\mathrm{T}$ is determined as 0.98 .

\section{EXPERIMENTAL RESULTS AND ANALYSIS}

In order to verify the validity of the proposed method for the change detection, the processing speed and the detection effect were tested respectively.

\subsection{Outline}

The camera is fixed in a certain location in the laboratory, and the videos are taken in four directions of the laboratory. DE1.1 infrared plane radar scans the indoor scene, getting two sets of data. After obtaining the data, the frame rate of the video data is set to 14 frames per second, and the acquisition rate of radar data is set to 14 cycles per second. There is a total of 73 change points in dataset 1 and 39 in dataset 2 .

\subsection{Speed Test}

Then, the classical hybrid Gauss modeling method proposed by Stauffer uses two kinds of data training background models and their processing speed are compared. Table 3 shows that the average speed of the background model is $27.4 \mathrm{~ms} / \mathrm{f}$ for training four sets of video data, while the average speed of training radar data is $19.1 \mathrm{~ms} / \mathrm{T}$.

\subsection{Detection Effect Test}

Adaptively Splitted GMM With Feedback Improvement for the Task of Background Subtraction (ASGMM) proposed by Evangelio is used to experiment on the four groups of video data respectively. The proposed method is used to experiment on the radar data. Finally, the ASGMM method and this method are both used to detect the radar data, we use recall, precision and $F$ to evaluate the performance of the change detection method here. They are calculated as follows:

$$
\begin{gathered}
\text { Recall }=\frac{T P}{T P+F N} \\
\text { Precision }=\frac{T P}{T P+F P} \\
F=2 /\left(\frac{1}{\text { Recall }}+\frac{1}{\text { Precision }}\right)
\end{gathered}
$$

Where TP, FN represent the number of correctly detected and undetected change objects, FP represents the number of falsely detected change targets and TN represents the number of correctly detected unchanging objects in the results.

As what we can see in figure 4 , if the detection scene is simple with less people and better illumination condition, such as scene 1 and scene 2 , the detection is relatively accurate, although there are still some error-detected objects. While, in the scene with more people and worth light condition, the detection results are 
Scene 1 \& Scene 2

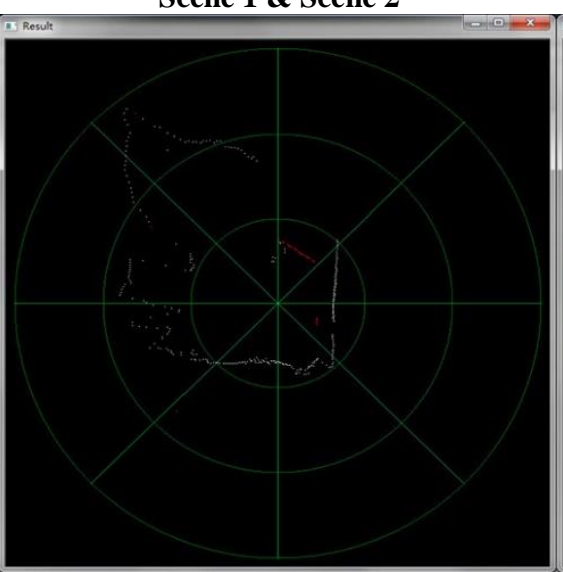

Scene 3

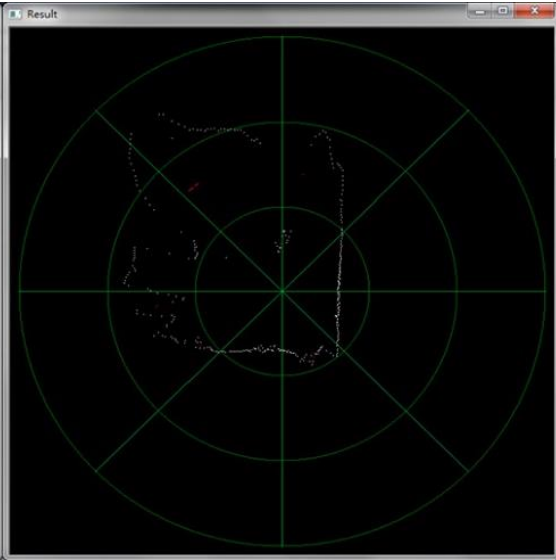

Figure 5. Detection result of the proposed method
Scene 4

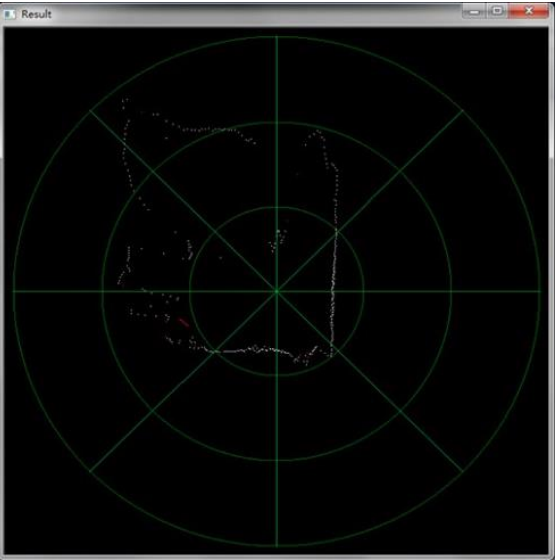

\begin{tabular}{cccccccccc}
\hline \multirow{2}{*}{ Method } & Data & Change Truth & TP & FP & TN & FN & $\begin{array}{c}\text { Recall } \\
(\%)\end{array}$ & $\begin{array}{c}\text { Precision } \\
(\%)\end{array}$ & F \\
\hline \multirow{2}{*}{ ASGMM } & Dataset1 & 73 & 65 & 7 & 268 & 8 & 89.0 & 90.3 & 89.7 \\
& Dataset2 & 39 & 34 & 6 & 277 & 5 & 87.2 & 85.0 & 86.1 \\
\multirow{2}{*}{ Proposed } & Dataset1 & 73 & 67 & 5 & 270 & 6 & 91.8 & 93.1 & 92.4 \\
& Dataset2 & 39 & 36 & 4 & 281 & 3 & 92.3 & 90.0 & 91.1 \\
\hline
\end{tabular}

Table 6. Quantitative results of change detection

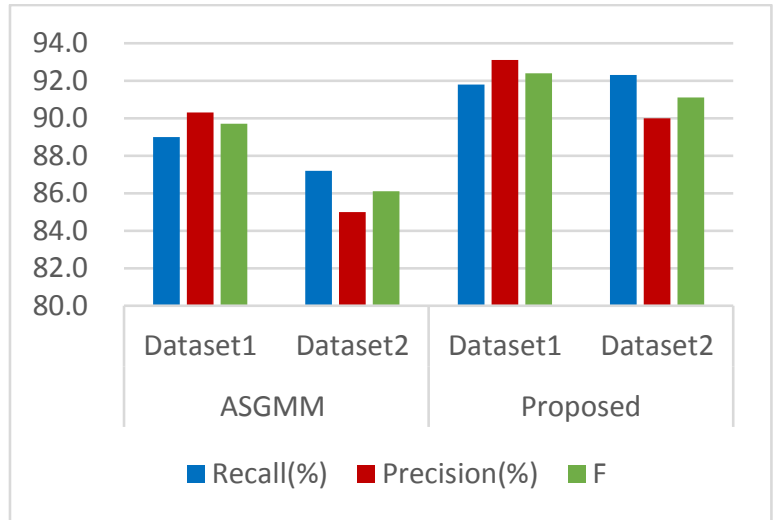

Figure 7. Change detection accuracy for each method

both with a large area of misdiagnosis. Figure 5 shows the detection results using the proposed method in this paper, because the scene 1 and the scene 2 appeared simultaneously, we can detect them at the same time. This proves that changes in multiple directions can be detected in a time, which shows the advantages of omni-directional scanning. As for scene 3 and scene 4 , the method can also provide accurate detection results, because that the infrared plane radar can detect objects without taking the illumination condition into account. The quantitative results are shown in table 6 . And figure 7 shows the change detection accuracy for each method.

\section{CONCLUSION}

This paper has discussed change detection based on infrared rotation plane radar. And the proposed method has been confirmed experimentally. The method easily solves the problems that affect most traditional method, such as the susceptibility to light, and the complex calculation and analysis for each pixel. This method has been improved in the speed of change detection and detection effect as well.
Only a cross section data of the scene is got in this paper, though this method is not affected by illumination and it can detect the change area effectively, it is not sure what has changed. In our future work, more than one set of data of different cross section will be used to get the shapes of the change targets.

\section{ACKNOWLEDGEMENTS}

This work was partially supported by The National Natural Science Foundation of China, Nos. 41561082, 41161061.

\section{REFERENCES}

Akula, A., Khanna, N., Ghosh, R., Kumar, S., Das, A., and Sardana, hk., 2014a. Adaptive contour-based statistical background subtraction method for moving target detection in infrared video sequences. Infrared Physics \& Technology, 63(11), pp. 103-109.

Bharti., and Thind, T., 2013a. Background Subtraction 
Techniques-Review. International Journal of Innovative Technology and Exploring Engineering, 2(3), pp. 3099-3104.

Evangelio, RH., Patzold, M., Keller, I., and Sikora, T., 2014a. Adaptively Splitted GMM With Feedback Improvement for the Task of Background Subtraction. IEEE Transactions on Information Forensics \& Security, 9(5), pp. 863-874

Huang, Xinjuan., Zhou, Jiemin., and Liu, Boyang., 2010a. Moving objects detection approach based on adaptive mixture Gaussian background model. Journal of Computer Applications, 30(1), pp. 71-74.

Hou, Jie., and Xin Yunhong., 2013a. A Method for Infrared Small Target Detection with High-pass Filter and Image Enhancement Technology. Infrared Technology, 35(5), pp. 279-284.

Li, Zhuo, and Qiu, Huijuan., 2007a. Fast Image Matching Based on Correlation Coefficient. Transactions of Beijing Institute of Technology, 27(10), pp. 998-1000.

Liu, Xiaochun., Yu, Qifeng., Lei, Zhihui., and Hou Wang, 2012a. Dim changing target detection of outside stereo scene for airborne platform. Journal of National University of Defense Technology, 34(6), pp. 130-135.

Power, PW., and Schoonees, JA., 2002a. Understanding Background Mixture Models for Foreground Segmentation. Imaging \& Vision Computing, pp. 267-271.
Radke, R.J., Andra, S., Al-Kofahi, O., and Roysam, B., 2005a. Image Change Detection Algorithms:A Systematic Survey. IEEE Transactions On Image Processing, 14(3), pp. 294-307.

Shimada, A., Nagahara, H., and Taniguchi, RI. 2016a. Background light ray modeling for change detection. Academic Press, 38(9), pp. 55-64

Stauffer, C., and Grimson, WEL., 1999a. Adaptive background mixture models for real-time tracking. International Conference on Computer Vision and Pattern Recognition, pp. 2246.

Sun, Peiqiang., 2013a. Correct selection of statistical discriminant method to eliminate abnormal values. Measuring Technique, 2013(11), pp. 71-73.

Wang, Xia., Liu, Zhenhua., Yu, Junpeng., and Deng, Chuqiang., 2014a. SAR Image Change Detection Based on Gaussian Mixture Model. Modem Radar, 36(9): 34-35.

Zhen, Jin., and Li, Bo., 2008a. Prospects and current studies on motion object detection in video sequences. Application Research of Computers, 25(12), pp. 3534-3540.

Zhou, Hong., Chen, Yiru., and Feng, Rong., 2013a. A novel background subtraction method based on color invariants. Computer Vision and Image Understanding, 117(11), pp. 15891597. 